\title{
US GEOLOGICAL SURVEY, DENVER, COLORADO RADIOCARBON DATES V
}

\author{
I C YANG \\ US Geological Survey, Denver, Colorado 80225 \\ INTRODUCTION
}

This list contains the results of measurements of 172 radiocarbon samples made between January 1983 and December 1985. An additional 152 samples were measured, but these were submitted without detailed information, such as sample location and sampling depth; therefore, they were excluded from the list.

${ }^{14} \mathrm{C}$ ages were computed using the radiocarbon half-life of $5568 \pm 30$ years. Statistical errors quoted herein are $1 \sigma$ counting errors, including sample, background, and standard counting rates. The age limit reported is calculated on the basis of $3 \sigma$ activity. The $\delta^{13} \mathrm{C}$ values in Table 1 were measured in an isotope laboratory of the US Geological Survey in Menlo Park, California, and calculated relative to Craig's Peedee Belemnite (PDB) limestone standard (Craig, 1957). Total alkalinity as bicarbonate values reported in Table 1 was determined using techniques described by Brown, Skougstad and Fishman (1970). Unless otherwise stated, all samples were collected and submitted by personnel of the US Geological Survey. Sample preparation and counting technique remain as previously reported (Yang \& Emerson, 1980; Yang, McAvoy \& Emerson, 1981; Yang, 1984). Most of the samples from Nevada were collected as part of the Nevada Nuclear Waste Storage Investigations conducted in cooperation with the US Department of Energy, Nevada Operations Office, under Interagency Agreement DE-AI08-78ET44802.

\section{GROUNDWATER SAMPLES}

United States

Arkansas

DE-236. Well near Piggot $\mathrm{W})$.

Sample coll July 26,1984 , from Clay Co $\left(36^{\circ} 22^{\prime} 25^{\prime \prime} \mathrm{N}, 090^{\circ} 12^{\prime} 08^{\prime \prime}\right.$

DE-237. Well 19NO4E01BDB 1

$39,900 \pm 1700$

Sample coll Aug 1, 1984, from Clay Co $\left(36^{\circ} 19^{\prime} 09^{\prime \prime} \mathrm{N}, 090^{\circ} 35^{\prime} 59^{\prime \prime} \mathrm{W}\right)$. Alt of well head, $85.3 \mathrm{~m}$ asl.

Califormia

Franklin Lake series

DE-238. Well 5

$28,700 \pm 840$

Sample coll Nov 21, 1983, from Franklin Lake near Death Valley Junction, Inyo Co $\left(36^{\circ} 14^{\prime} 06^{\prime \prime} \mathrm{N}, 116^{\circ} 17^{\prime} 32^{\prime \prime} \mathrm{W}\right)$. Hole drilled to $10.7 \mathrm{~m}$. 
DE-239. Well 10

$13,100 \pm 150$

Sample coll Nov 21, $1983\left(36^{\circ} 14^{\prime} 06^{\prime \prime}\right.$ N, $116^{\circ} 17^{\prime} 39^{\prime \prime}$ W). Hole drilled to $12.2 \mathrm{~m}$.

DE-240. Well GS-8

$12,350 \pm 180$

Sample coll Nov 19, $1983\left(36^{\circ} 17^{\prime} 27^{\prime \prime}\right.$ N, $116^{\circ} 17^{\prime} 09^{\prime \prime}$ W). Hole drilled to $10.1 \mathrm{~m}$.

DE-241. Well 15

$5800 \pm 140$

Sample coll Nov 21, $1983\left(36^{\circ} 18^{\prime} 51^{\prime \prime} \mathrm{N}, 116^{\circ} 17^{\prime} 00^{\prime \prime} \mathrm{W}\right)$.

DE-242. Well No. 14

$25,600 \pm 530$

Sample coll July 25, 1985, from Inyo Co $\left(36^{\circ} 14^{\prime} 15^{\prime \prime} \mathrm{N}, 116^{\circ} 23^{\prime} 00^{\prime \prime}\right.$ W). Static water level, $1.4 \mathrm{~m}$ below land surface.

DE-243. Well No. 16

$5900 \pm 130$ W).

Sample coll July 29 , 1985, from Inyo Co $\left(36^{\circ} 18^{\prime} 00^{\prime \prime} \mathrm{N}, 116^{\circ} 17^{\prime} 30^{\prime \prime}\right.$

DE-244. Charcoal

$1100 \pm 90$

Sample coll July 13, 1985, from Gravel Ck Valley, sec 36, NE $1 / 4, \mathrm{~T} 42$ N, R 3 W.

DE-245. Charcoal

$550 \pm 90$ R 2 W.

Sample coll July 10, 1984, from lower Gravel Ck, sec 21, SW $1 / 4, \mathrm{~T} 42 \mathrm{~N}$,

DE-246. Charcoal

$2300 \pm 900$

Sample coll July 10, 1984, from Mt Shasta fluvial deposits, sec $21 \mathrm{SE}^{1 / 4}$, T 42 N, R 2 W. Sample depth 1.6m.

DE-247. Charcoal

Modern

Sample coll July 13, 1984, from debris-flow deposit in Gravel Ck Valley near Mt Shasta, sec 31 NW11/4, T 42 N, R 2 W.

Colorado

DE-248. Well S(B-7-103) 32ADB-1

$10,500 \pm 220$ W).

Sample coll Aug 31, 1983, from Moffat Co $\left(40^{\circ} 31^{\prime} 08^{\prime \prime} \mathrm{N}, 108^{\circ} 59^{\prime} 19^{\prime \prime}\right.$

DE-249. Well SC00609417CAD1

$34,300 \pm 1400$ W).

Sample coll Oct 28, 1983, from Moffat Co $\left(40^{\circ} 28^{\prime} 03^{\prime \prime} \mathrm{N}, 107^{\circ} 57^{\prime} 10^{\prime \prime}\right.$

\section{Rio Blanco series}

Samples coll March 24, 1983, from Rio Blanco Co $\left(39^{\circ} 48^{\prime} 26^{\prime \prime} \mathrm{N}, 108^{\circ}\right.$ $13^{\prime} 28^{\prime \prime} \mathrm{W}$ ).

DE-250. Well SC00309712ABD1

$12,400 \pm 260$

Sample coll at depth $203.9 \mathrm{~m}$. 
DE-251. Well SC00309712ABDZ

Sample coll at depth $356.6 \mathrm{~m}$.

$20,700 \pm 730$

DE-252. Well SC00309712ABD3

Sample coll at depth $402.3 \mathrm{~m}$.

$21,600 \pm 760$

DE-253. Well SC00309712ABD4

$23,200 \pm 810$

Sample coll at depth $451.1 \mathrm{~m}$.

DE-254. Well SC00309712ABD5

$20,500 \pm 720$

Sample coll at depth $487 \mathrm{~m}$.

DE-255. Well SC00309712ACB1

Sample coll $\left(39^{\circ} 48^{\prime} 28^{\prime \prime} \mathrm{N}, 108^{\circ} 13^{\prime} 27^{\prime \prime} \mathrm{W}\right)$ at depth $445 \mathrm{~m}$.

DE-256. Well SC00209736ACA1

$5300 \pm 110$ to $22.7 \mathrm{~m}$.

Sample coll Oct 9, $1983\left(39^{\circ} 50^{\prime} 10^{\prime \prime} \mathrm{N}, 108^{\circ} 13^{\prime} 29^{\prime \prime} \mathrm{W}\right)$. Hole drilled

DE-257. Well SC00209736ABC2

$19,300 \pm 400$

Sample coll Oct 10, $1983\left(39^{\circ} 50^{\prime} 13^{\prime \prime} \mathrm{N}, 108^{\circ} 13^{\prime} 31^{\prime \prime} \mathrm{W}\right)$.

DE-258. Well SC00209803ABD TH75-11A

$26,100 \pm 910$

Sample coll Aug 26, $1983\left(39^{\circ} 54^{\prime} 39^{\prime \prime}\right.$ N, $108^{\circ} 22^{\prime} 33^{\prime \prime}$ W). Hole drilled to $356.6 \mathrm{~m}$. Alt of well head, $2039.4 \mathrm{~m}$ asl.

DE-259. Well TH 75-11 B (C2-98-30 DBA2) $\quad$ 29,000 \pm 1000

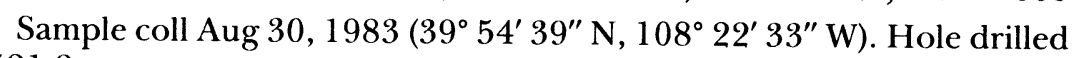
to $521.2 \mathrm{~m}$.

DE-260. Well TH 75-68 (Cl-98-14 ADC2) $\quad 30,000 \pm 1300$

Sample coll Oct 6, $1983\left(39^{\circ} 57^{\prime} 55^{\prime \prime} \mathrm{N}, 108^{\circ} 21^{\prime} 14^{\prime \prime} \mathrm{W}\right)$. Hole drilled to $533.4 \mathrm{~m}$.

DE-261. Well SC00109614ADCD2

$34,900 \pm 1500$

Sample coll Oct 4, $1983\left(39^{\circ} 57^{\prime} 55^{\prime \prime} \mathrm{N}, 108^{\circ} 21^{\prime} 14^{\prime \prime} \mathrm{W}\right)$. Hole drilled to $533.4 \mathrm{~m}$.

DE-262. Spring SC00400907DCCD1

$9500 \pm 200$

Sample coll July 18, $1983\left(39^{\circ} 42^{\prime} 36^{\prime \prime} \mathrm{N}, 108^{\circ} 32^{\prime} 40^{\prime \prime} \mathrm{W}\right)$.

DE-263. Spring SC00409910DBA1

$6200 \pm 130$

Sample coll Aug 22, 1983 ( $\left.39^{\circ} 42^{\prime} 56^{\prime \prime} \mathrm{N}, 108^{\circ} 29^{\prime} 10^{\prime \prime} \mathrm{W}\right)$.

DE-264. Spring SC00209736ACB1 (P-16)

$6000 \pm 130$

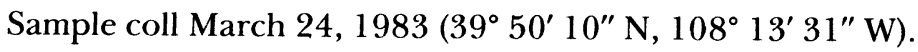


DE-265. Well SB00509830DBD-1

Sample coll Feb 7, 1984, from Moffat Co $\left(40^{\circ} 06^{\prime} 12^{\prime \prime} \mathrm{N}, 108^{\circ} 26^{\prime} 30^{\prime \prime}\right.$ W). Static water level, $204.2 \mathrm{~m}$ below land surface.

DE-266. Redstone Corp Well

$25,300 \pm 640$ $\left.04^{\prime \prime} \mathrm{W}\right)$

Sample coll Sept 13, 1984, from Garfield Co $\left(39^{\circ} 33^{\prime} 13^{\prime \prime}\right.$ N, $107^{\circ} 20^{\prime}$

Iowa

DE-290. Well 07227W10CDCA 211351968 Town of Murray >39,900

Sample coll July 10, 1984, from Clarke Co $\left(41^{\circ} 01^{\prime} 35^{\prime \prime} \mathrm{N}, 93^{\circ} 56^{\prime} 49^{\prime \prime}\right.$ W). Hole drilled to $932.7 \mathrm{~m}$. Alt of well head, $371.8 \mathrm{~m}$ asl.

DE-291. Well 07623W31DADD 237021976 Indianola City >39,900 No. 11

Sample coll July 11,1984 , from Warren Co $\left(41^{\circ} 20^{\prime} 25^{\prime \prime}\right.$ N, $93^{\circ} 32^{\prime} 22^{\prime \prime}$ W). Hole drilled to $740.7 \mathrm{~m}$. Alt of well head, $258 \mathrm{~m}$ above asl.

DE-292. Well 08321W08ACCA 109341959 Town of Colo >38,800 No. 3

Sample coll July 9, 1984, from Story Co $\left(42^{\circ} 00^{\prime} 59^{\prime \prime}\right.$ N, $\left.93^{\circ} 19^{\prime} 03^{\prime \prime}\right)$. Hole drilled to $236.2 \mathrm{~m}$. Alt of well head, $312.4 \mathrm{~m}$ above asl.

DE-293. Well 08721W33ABCB $021621945 \quad 8700 \pm 100$ Hubbard Town Well No. 2

Sample coll July 7, 1984, from Hardin Co (42 $18^{\prime} 33^{\prime \prime} 17^{\prime} 56^{\prime \prime}$ W). Hole drilled to $146.3 \mathrm{~m}$. Alt of well head, $333.5 \mathrm{~m}$ asl.

DE-294. Well 09114W03CABB Waverly 5 $2400 \pm 70$

Sample coll July 11,1984 , from Bremer Co $\left(42^{\circ} 43^{\prime} 19^{\prime \prime} \mathrm{N}, 92^{\circ} 28^{\prime} 34^{\prime \prime}\right.$ W). Hole drilled to $47.8 \mathrm{~m}$.

DE-295. Well 09114W02BADC 00055 Waverly 2

Sample coll July 11, 1984, from Bremer Co $\left(42^{\circ} 43^{\prime} 37^{\prime \prime} \mathrm{N}, 92^{\circ} 28^{\prime} 03^{\prime \prime}\right.$ W). Hole drilled to $384 \mathrm{~m}$. Alt of well head, $279.5 \mathrm{~m}$ asl.

DE-296. Well 09221 W31DBBD 1939 Coulter Iowa No. $1 \quad 6900 \pm 80$

Sample coll July 6, 1984, from Franklin Co $\left(42^{\circ} 44^{\prime} 13^{\prime \prime}\right.$ N, $93^{\circ} 22^{\prime} 06^{\prime \prime}$ W). Hole drilled to $191.4 \mathrm{~m}$. Alt of well head, $379.2 \mathrm{~m}$ asl.

DE-297. Well 09420W03ABCC 138381962 Rockwell Town $2>39,900$

Sample coll July 6, 1984 , from Cerro Gordo Co $\left(42^{\circ} 59^{\prime} 23^{\prime \prime}\right.$ N, $93^{\circ} 11^{\prime}$ $\left.06^{\prime \prime} \mathrm{W}\right)$. Hole drilled to $139.9 \mathrm{~m}$. Alt of well head, $346.6 \mathrm{~m}$ asl.

\section{DE-298. Well 09513W12ABDD 159361964 \\ $38,800 \pm 3800$ New Hampton No. 5}

Sample coll July 5, 1984, from Chickasaw Co $\left(43^{\circ} 3^{\prime} 49^{\prime \prime}\right.$ N, $92^{\circ} 19^{\prime} 23^{\prime \prime}$ W). Hole drilled to $402.3 \mathrm{~m}$. Alt of well head, $353.6 \mathrm{~m}$ asl. 
DE-299. Well 09516W01AAB 048691950 Charles City $6600 \pm 90$ Well No. 5

Sample coll July 5, 1984, from Floyd Co $\left(43^{\circ} 04^{\prime} 58^{\prime \prime} \mathrm{N}, 92^{\circ} 40^{\prime} 37^{\prime \prime} \mathrm{W}\right)$. Hole drilled to $57 \mathrm{~m}$. Alt of well head, $308.7 \mathrm{~m}$ asl.

DE-300. Well 09608W10ADDC 138421962 Ossian

$\mathbf{2 7 , 4 0 0} \pm \mathbf{7 7 0}$

Sample coll July 3, 1984, from Winneshiek Co $\left(43^{\circ} 08^{\prime} 52^{\prime \prime} \mathrm{N}, 91^{\circ} 45^{\prime}\right.$ $\left.59^{\prime \prime} \mathrm{W}\right)$. Hole drilled to $307.8 \mathrm{~m}$. Alt of well head, $387.1 \mathrm{~m}$ asl.

\section{DE-301. Well 09709W19CADA 117551960 Spillville}

$1500 \pm 70$

Sample coll July 3, 1984, from Winneshiek Co $\left(43^{\circ} 12^{\prime} 09^{\prime \prime}\right.$ N, $91^{\circ} 57^{\prime}$ $\left.16^{\prime \prime} \mathrm{W}\right)$. Hole drilled to $110.3 \mathrm{~m}$. Alt of well head, $324.9 \mathrm{~m}$ asl.

DE-302. Well 098-05W-30ACC2 Waukon Creamery No. $32800 \pm 70$ $\left.41^{\prime \prime} \mathrm{W}\right)$.

Sample coll July 3, 1984, from Allamakee Co $\left(43^{\circ} 16^{\prime} 38^{\prime \prime} \mathrm{N}, 91^{\circ} 28^{\prime}\right.$

DE-303. Well 10020W29DDDC 1931 Northwood No. $2 \quad 3800 \pm 70$

Sample coll July 6, 1984, from Worth Co $\left(43^{\circ} 26^{\prime} 42^{\prime \prime}\right.$ N, $93^{\circ} 13^{\prime} 21^{\prime \prime}$ W). Hole drilled to $49.3 \mathrm{~m}$. Alt of well head, $374.9 \mathrm{~m}$ asl.

DE-304. Well West Upton-Jordan WFCC

$>37,700$ W).

Sample coll July 12, 1984, from Fayette Co $\left(42^{\circ} 57^{\prime} 23^{\prime \prime}\right.$ N, $91^{\circ} 48^{\prime} 44^{\prime \prime}$

DE-305. Well Collins-Jordan

$>\mathbf{3 8 , 9 0 0}$ W).

Sample coll July 9, 1984, from Story Co $\left(41^{\circ} 54^{\prime} 04^{\prime \prime} \mathrm{N}, 93^{\circ} 18^{\prime} 11^{\prime \prime}\right.$

Missouri

Dunklin Co series

Samples from Dunklin Co.

DE-306. Well T16N R09E 08ACD1

$\mathbf{3 9 , 9 0 0} \pm \mathbf{4 5 0 0}$

Sample coll Aug 3, $1984\left(36^{\circ} 02^{\prime} 25^{\prime \prime} \mathrm{N}, 90^{\circ} 06^{\prime} 40^{\prime \prime} \mathrm{W}\right)$. Hole drilled to $560.8 \mathrm{~m}$. Alt of well head, $75 \mathrm{~m}$ asl.

DE-307. Well Cardwell \#3

$>\mathbf{3 9 , 9 0 0}$

Sample coll Aug 1, $1984\left(36^{\circ} 02^{\prime} 25^{\prime \prime} \mathrm{N}, 90^{\circ} 18^{\prime} 15^{\prime \prime} \mathrm{W}\right)$. Hole drilled to $499.9 \mathrm{~m}$. Alt of well head, $75.3 \mathrm{~m}$ asl.

DE-308. Well T18N R10E 02AAD1

$>39,900$

Sample coll July $30,1984\left(36^{\circ} 14^{\prime} 05^{\prime \prime} \mathrm{N}, 90^{\circ} 03^{\prime} 15^{\prime \prime} \mathrm{W}\right)$. Hole drilled to $463.3 \mathrm{~m}$. Alt of well head, $80.8 \mathrm{~m}$ asl.

DE-321. Well Holcomb T20N R10E 06BDD1 $>\mathbf{3 8 , 1 0 0}$

Sample coll July $26,1984\left(36^{\circ} 24^{\prime} 18^{\prime \prime} \mathrm{N}, 90^{\circ} 01^{\prime} 31^{\prime \prime} \mathrm{W}\right)$. Hole drilled to $378.0 \mathrm{~m}$. Alt of well head, $84.1 \mathrm{~m}$ asl. 
DE-322. Well Senath No. 2

Sample coll July $30,1984\left(36^{\circ} 08^{\prime} 11^{\prime \prime} \mathrm{N}, 90^{\circ} 09^{\prime} 49^{\prime \prime} \mathrm{W}\right)$. Hole drilled to $521.2 \mathrm{~m}$.

\section{New Madrid Co series}

Samples from New Madrid Co.

DE-309. Well City of Marston

$$
19,200 \pm 400
$$

Sample coll Nov 2, $1983\left(36^{\circ} 31^{\prime} 07^{\prime \prime} \mathrm{N}, 89^{\circ} 36^{\prime} 34^{\prime \prime} \mathrm{W}\right)$. Hole drilled to $502.9 \mathrm{~m}$. Alt of well head, $87.8 \mathrm{~m}$ asl.

DE-310. Well City of Marston

Sample coll Aug 2, $1984\left(36^{\circ} 31^{\prime} 07^{\prime \prime}\right.$ N, 89 $\left.36^{\prime} 34^{\prime \prime} \mathrm{W}\right)$. Hole drilled to $502.9 \mathrm{~m}$. Alt of well head, $87.8 \mathrm{~m}$ asl.

DE-312. Well City of Gideon

$$
27,600 \pm 970
$$

Sample coll Oct $13,1983\left(36^{\circ} 27^{\prime} 05^{\prime \prime} \mathrm{N}, 89^{\circ} 54^{\prime} 50^{\prime \prime} \mathrm{W}\right)$. Hole drilled to $399.2 \mathrm{~m}$. Alt of well head, $80.8 \mathrm{~m}$ asl.

\section{DE-313. Well City of Risco}

Sample coll Oct $27,1983\left(36^{\circ} 33^{\prime} 09^{\prime \prime} \mathrm{N}, 89^{\circ} 49^{\prime} 20^{\prime \prime} \mathrm{W}\right)$. Hole drilled to $353.6 \mathrm{~m}$. Alt of well head, $84.1 \mathrm{~m}$ asl.

DE-314. Well City of Parma No. 1

$\mathbf{5 9 0 0} \pm \mathbf{8 0}$

Sample coll Sept 26, 1984 ( $36^{\circ} 36^{\prime} 45^{\prime \prime}$ N, $\left.89^{\circ} 49^{\prime} 05^{\prime \prime} \mathrm{W}\right)$. Hole drilled to $144.8 \mathrm{~m}$. Alt of well head, $86.3 \mathrm{~m}$ asl.

DE-311. Well Fred Scherer, Bell City

$27,400 \pm 4400$

Sample coll July 31 , 1984, from Stoddard Co $\left(36^{\circ} 55^{\prime} 29^{\prime \prime} \mathrm{N}, 89^{\circ} 44^{\prime}\right.$ $\left.38^{\prime \prime} \mathrm{W}\right)$. Hole drilled to $121.9 \mathrm{~m}$. Alt of well head, $97.5 \mathrm{~m}$ asl.

\section{Pemiscot Co series}

Samples from Pemiscot Co.

DE-315. Well Steele No. 2-Deep well

Sample coll Sept 25, $1984\left(36^{\circ} 49^{\prime} 55^{\prime \prime}\right.$ N, $\left.89^{\circ} 49^{\prime} 58^{\prime \prime} \mathrm{W}\right)$. Hole drilled to $710.2 \mathrm{~m}$. Alt of well head, $79.2 \mathrm{~m}$ asl.

DE-316. Artesian Well-Hayti No. 3

$35,500 \pm 1500$

Sample coll Oct 12, $1983\left(36^{\circ} 14^{\prime} 18^{\prime \prime} \mathrm{N}, 89^{\circ} 45^{\prime} 02^{\prime \prime} \mathrm{W}\right)$. Hole drilled to $655.3 \mathrm{~m}$. Alt of well head, $82.3 \mathrm{~m}$ asl.

DE-317. Artesian Well-Hayti No. 3

Sample coll Oct $14,1983\left(36^{\circ} 16^{\prime} 00^{\prime \prime} \mathrm{N}, 89^{\circ} 49^{\prime} 50^{\prime \prime} \mathrm{W}\right)$. Hole drilled to $655.3 \mathrm{~m}$. Alt of well head, $82.3 \mathrm{~m}$ asl. 
DE-318. Well Pemiscot PWSD 2 at Pascola

$31,000 \pm 1300$

Sample coll Oct $12,1983\left(36^{\circ} 16^{\prime} 00^{\prime \prime} \mathrm{N}, 89^{\circ} 49^{\prime} 50^{\prime \prime} \mathrm{W}\right)$. Hole drilled to $591.3 \mathrm{~m}$. Alt of well head, $80.5 \mathrm{~m}$ asl.

DE-324. Well Hayti No. 7

$20,200 \pm 450$

Sample coll Sept 25, $1984\left(36^{\circ} 14^{\prime} 18^{\prime \prime} \mathrm{N}, 89^{\circ} 45^{\prime} 02^{\prime \prime} \mathrm{W}\right)$.

DE-319. Well Sikeston No. 4

$6200 \pm 120$

Sample coll Sept 26, 1984, from Scott Co $\left(36^{\circ} 59^{\prime} 39^{\prime \prime}\right.$ N, 89 $35^{\prime} 28^{\prime \prime}$ W). Hole drilled to $114.3 \mathrm{~m}$. Alt of well head, $99.7 \mathrm{~m}$ asl.

DE-320. Well T28N R13E 18DBB1

$1200 \pm 90$

Sample coll Oct 25, 1983, from Scott Co $\left(37^{\circ} 05^{\prime} 00^{\prime \prime} \mathrm{N}, 89^{\circ} 39^{\prime} 01^{\prime \prime}\right.$ W). Hole drilled to $27.4 \mathrm{~m}$. Alt of well head, $102.1 \mathrm{~m}$ asl.

DE-323. Well Charleston No. 4

$6400 \pm 200$ $\left.57^{\prime \prime} \mathrm{W}\right)$

Sample coll Sept 27, 1984, from Mississippi Co ( $36^{\circ} 55^{\prime} 33^{\prime \prime} \mathrm{N}, 89^{\circ} 20^{\prime}$

Nevada

\section{Clarke Co series}

Samples from Clark Co.

DE-330. Spring, Sandstone Spring No. 1

$5600 \pm 120$

Sample coll June 25, 1985 (36 $03^{\prime} 47^{\prime \prime}$ N, $\left.115^{\circ} 28^{\prime} 09^{\prime \prime} \mathrm{W}\right)$.

DE-331. Well BLM Visitor's Center

$6200 \pm 130$

Sample coll June $30,1985\left(36^{\circ} 07^{\prime} 44^{\prime \prime} \mathrm{N}, 115^{\circ} 26^{\prime} 03^{\prime \prime} \mathrm{W}\right)$. Alt of well head, $1152.1 \mathrm{~m}$ asl.

DE-332. Red Spring

$3700 \pm 270$

Sample coll June $26,1985\left(36^{\circ} 08^{\prime} 40^{\prime \prime} \mathrm{N}, 115^{\circ} 25^{\prime} 10^{\prime \prime} \mathrm{W}\right)$. Alt of land surface, $1115.6 \mathrm{~m}$ asl.

DE-333. Manse Well

$6100 \pm 130$

Sample coll June $27,1985\left(36^{\circ} 09^{\prime} 17^{\prime \prime} \mathrm{N}, 115^{\circ} 53^{\prime} 42^{\prime \prime} \mathrm{W}\right)$. Alt of well head, $865.6 \mathrm{~m}$ asl.

DE-334. Well Sky Mt Resort

$$
1400 \pm 100
$$

Sample coll June 28, 1985 (36 $\left.16^{\prime} 13^{\prime \prime} \mathrm{N}, 115^{\circ} 34^{\prime} 44^{\prime \prime} \mathrm{W}\right)$.

DE-336. Spring, Dry Lake

$28,200 \pm 1100$

Sample coll July $1,1985\left(36^{\circ} 27^{\prime} 18^{\prime \prime} \mathrm{N}, 114^{\circ} 50^{\prime} 38^{\prime \prime} \mathrm{W}\right)$. Alt of land surface, $638.3 \mathrm{~m}$ asl. 
DE-342. White Rock Spring

$26,800 \pm 1400$

Sample coll June 26, 1985 (36 $\left.10^{\prime} 27^{\prime \prime} \mathrm{N}, 115^{\circ} 28^{\prime} 43^{\prime \prime} \mathrm{W}\right)$. Alt of land surface, $115.6 \mathrm{~m}$ asl.

\section{Nye Co series}

Samples from Nye Co.

DE-325. Well USW H-3

$13,700 \pm 130$

Sample coll March 13, 1984 (36 49' 42" N, $116^{\circ} 28^{\prime} 00^{\prime \prime}$ W). Hole drilled to $1219.2 \mathrm{~m}$.

DE-326. Well UE-25c No. 3

$14,900 \pm 160$

Sample coll May 9, $1984\left(36^{\circ} 49^{\prime} 47^{\prime \prime} \mathrm{N}, 116^{\circ} 25^{\prime} 43^{\prime \prime} \mathrm{W}\right)$. Hole drilled to $914.4 \mathrm{~m}$.

DE-335. Well Near Pahrump Spring

$10,800 \pm 230$

Sample coll June $27,1985\left(36^{\circ} 12^{\prime} 27^{\prime \prime} \mathrm{N}, 115^{\circ} 59^{\prime} 01^{\prime \prime} \mathrm{W}\right)$. Alt of well head, $823 \mathrm{~m}$ asl.

DE-337. Well USW H-3

$13,700 \pm 130$

Sample coll March 14, $1984\left(36^{\circ} 49^{\prime} 42^{\prime \prime}\right.$ N, $116^{\circ} 28^{\prime} 00^{\prime \prime}$ W). Static water level, $751 \mathrm{~m}$ below land surface. Also see sample DE-325.

DE-339. Well UE-25c No. 1

$15,200 \pm 320$

Sample coll Sept 30, $1983\left(36^{\circ} 49^{\prime} 47^{\prime \prime}\right.$ N, $116^{\circ} 25^{\prime} 43^{\prime \prime}$ W). Hole drilled to $914.4 \mathrm{~m}$.

DE-340. Well UE-25c No. 2

$15,100 \pm 210$

Sample coll March 13, $1984\left(36^{\circ} 49^{\prime} 47^{\prime \prime}\right.$ N, $116^{\circ} 25^{\prime} 43^{\prime \prime}$ W). Hole drilled to $914.4 \mathrm{~m}$.

DE-341. Duckwater Spring Railroad V

$27,900 \pm 980$

Sample coll June 13, $1983\left(38^{\circ} 57^{\prime} 01^{\prime \prime} \mathrm{N}, 115^{\circ} 42^{\prime} 06^{\prime \prime} \mathrm{W}\right)$. Alt of land surface, $1708.1 \mathrm{~m}$ asl.

DE-343. Fortymile Wash at well site J-13

$900 \pm 60$

Charcoal coll Dec 12, 1983 (36 48 34" N, $116^{\circ} 24^{\prime} 03^{\prime \prime}$ W). Sample from mud plaster on canyon wall, $1.2 \mathrm{~m}$ above stream bed.

DE-344. Well SP11 Fortymile Canyon

$1600 \pm 50$

Sample coll June 1, 1984 ( $\left.36^{\circ} 55^{\prime} 31^{\prime \prime} \mathrm{N}, 116^{\circ} 22^{\prime} 31^{\prime \prime} \mathrm{W}\right)$. Static water level, $18.0 \mathrm{~m}$ below land surface.

DE-345. Well USW H-6

$16,800 \pm 310$

Sample coll July 6, $1984\left(36^{\circ} 50^{\prime} 49^{\prime \prime} \mathrm{N}, 116^{\circ} 28^{\prime} 55^{\prime \prime} \mathrm{W}\right)$. Static water level, $627 \mathrm{~m}$ below land surface. 
DE-346. Fortymile Wash at well site J-13

Vegetal fragments from mud plaster on canyon wall coll June 20, 1984 $\left(36^{\circ} 48^{\prime} 34^{\prime \prime} \mathrm{N}, 116^{\circ} 24^{\prime} 03^{\prime \prime} \mathrm{W}\right), 1.2 \mathrm{~m}$ above stream bed.

DE-347. Well USW H-6

$18,500 \pm 220$

Sample coll June 20, 1984 ( $\left.36^{\circ} 50^{\prime} 49^{\prime \prime} \mathrm{N}, 116^{\circ} 28^{\prime} 55^{\prime \prime} \mathrm{W}\right)$. Static water level, $794 \mathrm{~m}$ below land surface.

DE-348. Busted Butte Wash

$850 \pm 150$

Surface water coll Aug 15, 1984 (36 $\left.48^{\circ} 25^{\prime \prime} \mathrm{N}, 116^{\circ} 23^{\prime} 39^{\prime \prime} \mathrm{W}\right)$.

DE-349. Fortymile Wash at Rd "H"

$1400 \pm 80$

Surface water coll Aug 15, 1984 (36 48 29" N, $116^{\circ} 23^{\prime} 34^{\prime \prime}$ W).

\section{White Pine Co series}

Samples from White Pine Co.

DE-327. Preston Big Spring

$15,900 \pm 330$

Sample coll June $16,1983\left(38^{\circ} 55^{\prime} 40^{\prime \prime} \mathrm{N}, 115^{\circ} 04^{\prime} 57^{\prime \prime} \mathrm{W}\right)$. Alt of land surface, $1737.4 \mathrm{~m}$ asl.

DE-328. 02ACAB1 Preston Big Spring

$17,600 \pm 830$

Sample coll June $26,1984\left(38^{\circ} 55^{\prime} 40^{\prime \prime} \mathrm{N}, 115^{\circ} 04^{\prime} 57^{\prime \prime} \mathrm{W}\right)$. Alt of land surface, $1737.4 \mathrm{~m}$ asl.

DE-329. 25CCCC1 Well at Alligator Ridge $\mathbf{3 3 , 2 0 0} \pm \mathbf{4 6 0 0}$

Sample coll April 24, $1984\left(39^{\circ} 44^{\prime} 27^{\prime \prime} \mathrm{N}, 115^{\circ} 30^{\prime} 43^{\prime \prime} \mathrm{W}\right)$. Hole drilled to $200.9 \mathrm{~m}$. Alt of well head, $2023.9 \mathrm{~m}$ asl.

DE-338. Amoco Expl Well Newark V

$14,600 \pm 250$

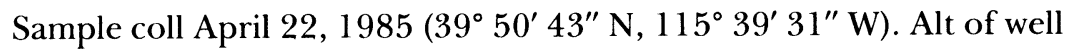
head, $1795.3 \mathrm{~m}$ asl.

Puerto Rico

DE-373. Well Sabana Hoyos No. 3 Arecibo $\quad 000 \pm 80$

Sample coll March 23, 1984, from Caguas Co $\left(18^{\circ} 24^{\prime} 21^{\prime \prime} \mathrm{N}, 66^{\circ} 36^{\prime}\right.$ $02 " \mathrm{~W}$ ). Hole drilled to $213.4 \mathrm{~m}$.

DE-374. Well Prasa Monte Encantado Moca

$1300 \pm 70$

Sample coll March 24, 1984, from Caguas Co (18 $24^{\prime} 58^{\prime \prime} \mathrm{N}, 67^{\circ} 02^{\prime}$ $\left.15^{\prime \prime} \mathrm{W}\right)$. Hole drilled to $61 \mathrm{~m}$.

DE-375. Well Prasa Sabana NR Pike

$8300 \pm 160$

Sample coll March 23, 1984, from Barceloneta Co $\left(18^{\circ} 25^{\prime} 04^{\prime \prime} \mathrm{N}, 66^{\circ}\right.$ $\left.35^{\prime} 01^{\prime \prime} \mathrm{W}\right)$. 
DE-376. Well Prasa Artesian Crule Davila $7900 \pm 90$

Sample coll March 20, 1984, from Cayey Co $\left(18^{\circ} 25^{\prime} 55^{\prime \prime} \mathrm{N}, 66^{\circ} 34^{\prime} 02^{\prime \prime}\right.$ W). Hole drilled to $374.9 \mathrm{~m}$.

DE-377. Zanja Fria Spring Arecibo $3800 \pm 80$

Sample coll March 27, 1984, from Caguas Co (18 $27^{\prime} 24^{\prime \prime} \mathrm{N}, 66^{\circ} 39^{\prime}$ $\left.20^{\prime \prime} \mathrm{W}\right)$.

DE-378. Well Garrochales No. 3 Barceloneta

$4800 \pm 70$

Sample coll March 23, 1984, from Cayey Co $\left(18^{\circ} 27^{\prime} 35^{\prime \prime} \mathrm{N}, 66^{\circ} 36^{\prime} 57^{\prime \prime}\right.$ W). Hole drilled to $24.4 \mathrm{~m}$.

DE-379. Pampanos Well

$6600 \pm 70$ W).

Sample coll May 1, 1984, from Vega Alta Co $\left(18^{\circ} 23^{\prime} 15^{\prime \prime} \mathrm{N}, 66^{\circ} 18^{\prime} 59^{\prime \prime}\right.$

DE-380. Well Prasa Florida No. 3

$3600 \pm 60$ $\left.40^{\prime \prime} \mathrm{W}\right)$

Sample coll March 26, 1984, from Arecibo Co $\left(18^{\circ} 21^{\prime} 50^{\prime \prime} \mathrm{N}, 66^{\circ} 35^{\prime}\right.$

DE-381. La Pollero Well

$\mathbf{5 0 0 0} \pm \mathbf{8 0}$

Sample coll March 23, 1984, from Barceloneta Co $\left(18^{\circ} 26^{\prime} 41^{\prime \prime} \mathrm{N}, 66^{\circ}\right.$ $\left.34^{\prime} 56^{\prime \prime} \mathrm{W}\right)$.

\section{South Carolina}

DE-382. Well BFT-210 Sea Pines Windmill

$30,200 \pm 3900$

Sample coll May 21, 1985, from Beaufort Co $\left(32^{\circ} 08^{\prime} 35^{\prime \prime} \mathrm{N}, 80^{\circ} 47^{\prime}\right.$ $22^{\prime \prime} \mathrm{W}$ ), at depth 39.6 to $48.8 \mathrm{~m}$. Alt of well head, $1.8 \mathrm{~m}$ asl.

DE-383. Well JAS-136 Co Rd 92

Sample coll June 26 , 1985, from Jasper Co $\left(32^{\circ} 09^{\prime} 08^{\prime \prime} \mathrm{N}, 80^{\circ} 59^{\prime} 49^{\prime \prime}\right.$ W), at depth 61.0 to $74.7 \mathrm{~m}$. Alt of well head, $6.1 \mathrm{~m}$ asl.

DE-384. Well BFT-439 Seapines South

Sample coll July 23, 1985, from Beaufort Co $\left(32^{\circ} 09^{\prime} 10^{\prime \prime} \mathrm{N}, 80^{\circ} 47^{\prime}\right.$ $20^{\prime \prime} \mathrm{W}$ ), at depth 55.5 to $59.4 \mathrm{~m}$. Alt of well head, $2.4 \mathrm{~m}$ asl.

DE-385. Well BFT-565 TH No. 4 Parris Islands

$16,400 \pm 950$

Sample coll May 22, 1985, from Beaufort Co $\left(32^{\circ} 19^{\prime} 23^{\prime \prime} \mathrm{N}, 80^{\circ} 40^{\prime}\right.$ $21^{\prime \prime} \mathrm{W}$ ), at depth 27.1 to $63.1 \mathrm{~m}$. Alt of well head, $3.3 \mathrm{~m}$ asl.

DE-386. Well HAM-122 Rd 20

$7900 \pm 350$

Sample coll June 25,1985 , from Hampton Co $\left(32^{\circ} 39^{\prime} 49^{\prime \prime} \mathrm{N}, 81^{\circ} 19^{\prime}\right.$ $30^{\prime \prime} \mathrm{W}$ ), at depth 25.0 to $53.0 \mathrm{~m}$. Alt of well head, $22.9 \mathrm{~m}$ asl. 
Tennessee

\section{Shelby Co series}

Samples from Shelby Co.

DE-387. Well SH:J-146 MLGW-DAVIS

$3800 \pm 70$

Sample coll Aug 22, $1984\left(35^{\circ} 01^{\prime} 14^{\prime \prime} \mathrm{N}, 90^{\circ} 07^{\prime} 17^{\prime \prime} \mathrm{W}\right)$. Hole drilled to $135.9 \mathrm{~m}$.

DE-388. Well SH:L-36 MLGW-LICHTERMAN $\quad 4400 \pm 100$

Sample coll Aug 24, $1984\left(35^{\circ} 02^{\prime} 18^{\prime \prime} \mathrm{N}, 89^{\circ} 51^{\prime} 17^{\prime \prime} \mathrm{W}\right)$. Hole drilled to $172.8 \mathrm{~m}$.

DE-389. Well SH:L-37 MLGW-LICHTERMAN $23,000 \pm 80$

Sample coll April 25, 1985 ( $\left.35^{\circ} 02^{\prime} 30^{\prime \prime} \mathrm{N}, 89^{\circ} 51^{\prime} 23^{\prime \prime} \mathrm{W}\right)$. Hole drilled to $116.4 \mathrm{~m}$.

DE-390. Well SH:K-74 SHEAHAN SOUTH WELL $1400 \pm 70$ FIELD

Sample coll April 25, 1985 (35 $\left.05^{\prime} 16^{\prime \prime} \mathrm{N}, 89^{\circ} 55^{\prime} 38^{\prime \prime} \mathrm{W}\right)$. Hole drilled to $83.2 \mathrm{~m}$.

DE-391. WELL SH:K-73 MLGW-SHEAHAN $1300 \pm \mathbf{6 0}$

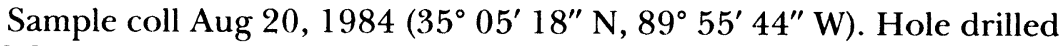
to $83.2 \mathrm{~m}$

DE-392. WELL SH:P-99 OVERTON PARK-2

$1900 \pm 60$ to $18 \mathrm{~m}$.

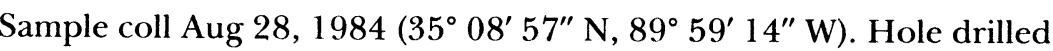

DE-393. SH:O-223 MALLORY WELL FIELD

$2100 \pm 60$

Sample coll April 26, 1985 ( $35^{\circ} 09^{\prime} 13^{\prime \prime}$ N, $90^{\circ} 00^{\prime} 58^{\prime \prime}$ W). Hole drilled to $235.3 \mathrm{~m}$.

DE-394. WELL SH:O-207 MLGW \#120

$1700 \pm 60$

Sample coll Aug 14, 1984 ( $35^{\circ} 09^{\prime} 13^{\prime \prime}$ N, $90^{\circ} 10^{\prime} 08^{\prime \prime}$ W). Hole drilled to $231 \mathrm{~m}$.

DE-395. WELL SH:P-23 BUCKEYE

$21,000 \pm 340$

Sample coll Aug 30, 1984 ( $\left.35^{\circ} 09^{\prime} 30^{\prime \prime} \mathrm{N}, 89^{\circ} 57^{\prime} 45^{\prime \prime} \mathrm{W}\right)$. Hole drilled to $429.8 \mathrm{~m}$.

DE-397. WELL SH:O-169 MLGW-MALLORY $\quad 39,900 \pm 1700$

Sample coll Oct $11,1983\left(35^{\circ} 09^{\prime} 08^{\prime \prime} \mathrm{N}, 90^{\circ} 01^{\prime} 46^{\prime \prime} \mathrm{W}\right)$. Hole drilled to $810.8 \mathrm{~m}$. 
DE-399. WELL AR:N-5

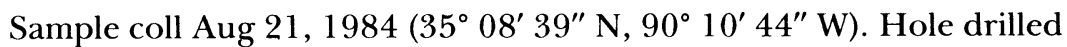
to $135.6 \mathrm{~m}$.

DE-396. WELL 20N08E15BAA1

Sample coll July 26 , 1984 , from Clay Co $\left(36^{\circ} 22^{\prime} 25^{\prime \prime} \mathrm{N}, 90^{\circ} 12^{\prime} 08^{\prime \prime} \mathrm{W}\right)$. Hole drilled to $323.1 \mathrm{~m}$. Alt of well head, $109.7 \mathrm{~m}$ asl.

DE-398. WELL HY:J-1 Paris, TN

$11,300 \pm 110$

Sample coll Aug 17, 1984, from Henry Co $\left(36^{\circ} 18^{\prime} 04^{\prime \prime}\right.$ N, 88 $18^{\circ} 45^{\prime \prime}$ W). Hole drilled to $161.2 \mathrm{~m}$. Alt of well head, $150.0 \mathrm{~m}$ asl.

Utah

\section{San Juan Co series}

Samples from San Juan Co.

DE-400. WELL (D-40-24) 17 DBD-1

$>\mathbf{3 7 , 9 0 0}$

Sample coll Oct $25,1984\left(37^{\circ} 18^{\prime} 30^{\prime \prime} \mathrm{N}, 109^{\circ} 17^{\prime} 50^{\prime \prime} \mathrm{W}\right)$. Hole drilled to $281.9 \mathrm{~m}$. Alt of well head, $139.6 \mathrm{~m}$ asl.

DE-401. WELL (D-39-25) 5ACA-2

$33,200 \pm 1700$

Sample coll June 19, 1984 (37 $\left.25^{\prime} 39^{\prime \prime} \mathrm{N}, 109^{\circ} 11^{\prime} 34^{\prime \prime} \mathrm{W}\right)$. Hole drilled to $396.2 \mathrm{~m}$. Alt of well head, 1450.8 asl.

DE-402. WELL (D-39-25) 5ACA-1

$\mathbf{2 4 , 0 0 0} \pm \mathbf{5 0 0}$

Sample coll June $19,1984\left(37^{\circ} 25^{\prime} 40^{\prime \prime} \mathrm{N}, 109^{\circ} 11^{\prime} 34^{\prime \prime} \mathrm{W}\right)$. Hole drilled to $113.4 \mathrm{~m}$. Alt of well head, $1452.7 \mathrm{~m}$ asl.

DE-403. WELL (D-38-25) 35 BDX-1

$32,300 \pm 1500$

Sample coll June $20,1984\left(37^{\circ} 26^{\prime} 28^{\prime \prime} \mathrm{N}, 109^{\circ} 08^{\prime} 38^{\prime \prime} \mathrm{W}\right)$. Alt of well head, $1478.3 \mathrm{~m}$ asl.

DE-404. WELL (D-37-24) 25BBD-1

$32,300 \pm 1500$

Sample coll June 6, $1984\left(37^{\circ} 32^{\prime} 50^{\prime \prime} \mathrm{N}, 109^{\circ} 14^{\prime} 20^{\prime \prime} \mathrm{W}\right)$. Hole drilled to $217 \mathrm{~m}$. Alt of well head, $1520.9 \mathrm{~m}$ asl.

DE-405. WELL (D-36-24) 14DBA-1

$22,700 \pm 560$

Sample coll June 7, 1984 ( $37^{\circ} 39^{\prime} 27^{\prime \prime}$ N, $109^{\circ} 14^{\prime} 46^{\prime \prime}$ W). Hole drilled to $74.7 \mathrm{~m}$. Alt of well head, $1639.8 \mathrm{~m}$ asl.

DE-406. WELL (D-35-24) 14CGA-1

$21,900 \pm 370$ W).

Sample coll June 7, 1984, from Utah Co $\left(37^{\circ} 42^{\prime} 00^{\prime \prime} \mathrm{N}, 109^{\circ} 16^{\prime} 00^{\prime \prime}\right.$

DE-407. WELL (D-38-25) 07DBB01

$27,200 \pm 740$ $\mathrm{W})$.

Sample coll June 21, 1984, from Utah Co (38 $29^{\prime} 47^{\prime \prime} \mathrm{N}, 109^{\circ} 12^{\prime} 46^{\prime \prime}$ 
SOIL SAMPLES

\section{United States}

Indiana

\section{Cowles Bog series}

Samples coll Oct 5, 1983, from Cowles Bog, Indiana Dunes Natl Lakeshore, Porter Co, sec 22, T 37 N, R 6 W (41 $38^{\prime}$ N, $\left.87^{\circ} 06^{\prime} \mathrm{W}\right)$.

\section{DE-267.}

$$
1000 \pm 70
$$

Fibrous peat; hole drilled to 0.30 to $0.50 \mathrm{~m}$ below peat surface.

DE-268.

$2000 \pm 80$

Fibrous peat; hole drilled to 1.30 to $1.50 \mathrm{~m}$ below peat surface.

DE-269.

$3500 \pm 70$

Fibrous peat; hole drilled to 2.3 to $2.5 \mathrm{~m}$ below peat surface.

DE-270.

$5600 \pm 160$

Marly lake sediment; hole drilled to 3.6 to $3.7 \mathrm{~m}$ below peat surface.

DE-271.

$7800 \pm 450$ surface.

Marly and sandy lake sediment; hole drilled to 4.9 to $5.0 \mathrm{~m}$ below peat

DE-287.

$6000 \pm 230$

Marl mixed with organic particles coll Oct $5,1983\left(41^{\circ} 38^{\prime} 39^{\prime \prime} \mathrm{N}, 87^{\circ}\right.$ $06^{\prime} 40^{\prime \prime} \mathrm{W}$ ), at depth 4.5 to $4.65 \mathrm{~m}$.

DE-289.

Modern

Fibrous peat coll Aug 30, $1984\left(41^{\circ} 38^{\prime} 30^{\prime \prime} \mathrm{N}, 87^{\circ} 05^{\prime} 36^{\prime \prime} \mathrm{W}\right)$, at depth 5.6 to $5.8 \mathrm{~m}$.

\section{Miller Woods series}

Samples coll Oct 20, 1983, from Miller Woods, Indiana Dunes Natl Lakeshore, Lake Co, sec 1, T 36 N, R 8 W (41 $36^{\circ}$ N, $\left.87^{\circ} 17^{\prime} \mathrm{W}\right)$.

DE-272. Pond 56

$2400 \pm 70$

Sandy peat; hole drilled to 0.94 to $1.10 \mathrm{~m}$ below sediment surface.

DE-273. Pond 39

$2100 \pm 80$

Sandy peat; hole drilled to 1.25 to $1.40 \mathrm{~m}$ below sediment surface.

DE-274. Pond 53

$2700 \pm 70$

Sandy peat; hole drilled to 10.7 to $12.6 \mathrm{~m}$ below sediment surface.

DE-275. Pond 53

$1400 \pm 90$

Sandy peat coll Oct 19,1983 . Hole drilled to 0.60 to $0.77 \mathrm{~m}$ below sediment surface. 
DE-276. Pond 53

Sandy peat coll Oct 19,1983 . Hole drilled to 0.12 to $27 \mathrm{~m}$ below sediment surface.

DE-286. Pond 5

$2100 \pm 120$

Marly organic lake mud coll May 8, $1984\left(41^{\circ} 37^{\prime} 00^{\prime \prime} \mathrm{N}, 87^{\circ} 16^{\prime} 00^{\prime \prime}\right.$ W), at depth 0.85 to $0.90 \mathrm{~m}$.

DE-288. Pond 53

$3000 \pm 90$

Organic lake sediment mixed with sand coll May $8,1984\left(41^{\circ} 36^{\prime} 20^{\prime \prime}\right.$ $\mathrm{N}, 87^{\circ} 17^{\prime} 12^{\prime \prime} \mathrm{W}$ ), at depth 1.35 to $1.45 \mathrm{~m}$.

\section{Pinhook Bog series}

Samples coll Nov 1, 1983, from Pinhook Bog, Indiana Dunes Natl Lakeshore, La Porte Co, sec 35, T 37 N, R 4 W (41 $\left.31^{\circ} \mathrm{N}, 86^{\circ} 50^{\prime} \mathrm{W}\right)$.

DE-277.

$2400 \pm 80$

Fibrous peat; hole drilled to 2.15 to $2.4 \mathrm{~m}$ below peat surface.

DE-278.

$2200 \pm 90$

Fibrous peat; hole drilled to 1.45 to $1.65 \mathrm{~m}$ below peat surface.

DE-279.

$1000 \pm 70$

Fibrous peat; hole drilled to 0.65 to $0.80 \mathrm{~m}$ below peat surface.

DE-280.

$2200 \pm 70$

Fibrous woody peat coll at depth 5.49 to $5.69 \mathrm{~m}$. Comment: samples DE280 to -285 coll Oct $31,1983\left(41^{\circ} 37^{\prime} 00^{\prime \prime} \mathrm{N}, 86^{\circ} 51^{\prime} 41^{\prime \prime} \mathrm{W}\right)$.

DE-281.

Fibrous peat coll at depth 7.8 to $7.9 \mathrm{~m}$.

$4200 \pm 80$

DE-282.

$5500 \pm 90$

Fibrous peat coll at depth 9.14 to $9.29 \mathrm{~m}$.

DE-283.

$8300 \pm 100$

Fine organic detritus mixed with clay coll at depth 10.65 to $10.75 \mathrm{~m}$.

DE-284.

$10,500 \pm 150$

Clay mixed with fine organic detritus coll at depth 11.9 to $12.0 \mathrm{~m}$.

DE-285.

$1200 \pm 230$

Clay mixed with fine organic detritus coll at depth 12.95 to $13.05 \mathrm{~m}$.

Nevada

Walker Lake sediment series, Nye Co

All sample depths indicated are below water-sediment interface.

DE-350. WLC-4, seg 2, organic

$1600 \pm 190$

Sample coll Sept 7, 1984, from depth 0.60 to $0.69 \mathrm{~m}$. 
DE-351. WLC-4(3)-2, organic

Sample coll Sept 7, 1984, from depth 1.10 to $1.18 \mathrm{~m}$.

DE-352. WLC-4(4)-3, organic

Sample coll Sept 7, 1984, from depth 1.05 to $1.12 \mathrm{~m}$.

DE-353. WLC-4(5)-4, organic

Sample coll Sept 7, 1984, from depth 2.68 to $2.75 \mathrm{~m}$.

DE-354. WLC-4(7)-5, inorganic

$5400 \pm 250$ organic

Sample coll Sept 7, 1984, from depth 16.04 to $16.17 \mathrm{~m}$.

DE-355. WLC-4(9)-6, organic

$14,200 \pm 1300$

Sample coll Sept 7, 1984, from depth 22.35 to $22.45 \mathrm{~m}$.

DE-356. WLC-4(10)-7, organic

$15,700 \pm 680$

Sample coll Sept 7, 1984, from depth 24.60 to $24.67 \mathrm{~m}$.

DE-357. WLC-4(11)-8, organic

$16,800 \pm 1000$

Sample coll Sept 7, 1984, from depth 26.16 to $26.24 \mathrm{~m}$.

DE-358. WLC-4(12)-9, inorganic

$16,500 \pm 1000$ organic

Sample coll Sept 7, 1984, from depth 30.41 to $30.62 \mathrm{~m}$.

DE-359. WLC-4(13)-10, inorganic

$19,500 \pm 1000$ organic

$21,000 \pm 1100$

Sample coll Sept 7, 1984, from depth 32.48 to $32.62 \mathrm{~m}$.

DE-360. WLC-4(14)-11, inorganic

$26,700 \pm 1100$ organic

Sample coll Sept 7, 1984, from depth 35.09 to $35.18 \mathrm{~m}$.

DE-361. WLC-4(16)-12, organic

$30,900 \pm 6000$

Sample coll Sept 7, 1984, from depth 39.91 to $39.98 \mathrm{~m}$.

DE-362. WLC-4(17)-13, inorganic organic

$24,900 \pm 1900$

, from depth 42.36 to $42.43 \mathrm{~m}$.

DE-363. WLC-4(19)-4, organic

$>33,700$

Sample coll Sept 7, 1984, from depth 44.81 to $44.88 \mathrm{~m}$.

DE-364. WLC-5(5)-16, inorganic organic 
DE-365. WLC-5(6)-17, organic

$$
31,400 \pm 8400
$$

Sample coll Nov 30,1984 , from depth 39.71 to $39.83 \mathrm{~m}$.

DE-366. WLC-6(1)-18, inorganic

$$
350 \pm 100
$$

$$
\text { organic }
$$

Sample coll Nov 30, 1984, from depth 1.0 to $1.15 \mathrm{~m}$.

DE-367. WLC-8(2A)-19, inorganic

Sample coll Nov 30, 1984, from depth 2.83 to $2.99 \mathrm{~m}$.

DE-370. WLC-8(4)-20, inorganic

$$
\text { organic }
$$

Sample coll Nov 30, 1984, from depth 6.92 to $7.07 \mathrm{~m}$.

DE-371. WLC-8(8)-21, inorganic

Sample coll Nov 30,1984 , from depth 10.00 to $10.24 \mathrm{~m}$.

DE-368. WLC-4(8)-23, organic

Sample coll June 6, 1985, from depth 19.65 to $19.89 \mathrm{~m}$.

DE-369. WLC-8(9)-29, inorganic

Sample coll June 6, 1985, from depth 10.90 to $11.15 \mathrm{~m}$.

DE-372. WLC-8(2B)-30, inorganic

Sample coll June 6, 1985, from depth 4.23 to $4.39 \mathrm{~m}$.

\section{REFERENCES}

Brown, E, Skougstad, M W and Fishman, M J, 1970, US Geological Survey techniques of water-resources investigations: Book 5, Chap Al, p 42-44.

Craig, H, 1957, Isotopic standards for carbon and oxygen and factors for mass-spectrometric analysis of carbon dioxide: Geochim et Cosmochim Acta, v 12, p 133-149.

Yang, I C, 1984, US Geological Survey, Denver, Colorado, radiocarbon dates IV: Radiocarbon, v 26 , no. 2, p 166-184.

Yang, I C and Emerson, R L, 1980, Teflon vials for low-level ${ }^{14} \mathrm{C}$ liquid scintillation counting, in Peng, C T, Horrocks, D L and Alpen, E L, eds, Internatl conf on liquid scintillation counting, recent applications and developments: New York, Academic Press, v 2, p 181197

Yang, I C, McAvoy, R L and Emerson, R L, 1981, US Geological Survey, Denver, Colorado, radiocarbon dates III: Radiocarbon, $v 23$, no. 1, p 24-32. 
TABLE 1

Summary of $\delta^{13} \mathrm{C}$ and alkalinity for water sources

\begin{tabular}{|c|c|c|c|c|}
\hline $\begin{array}{l}\text { Sample } \\
\text { no. }\end{array}$ & $\begin{array}{c}\text { Colln } \\
\text { date }\end{array}$ & $\begin{array}{c}\delta^{13} \mathrm{C} \\
\left(\%{ }^{\circ} \mathrm{PDB}\right) \\
\text { Inorg } \\
\text { carbon }\end{array}$ & $\begin{array}{c}\text { Total } \\
\text { alkalinity } \\
\text { as } \\
\text { bicarbonate } \\
(\mathrm{mg} / \mathrm{L})\end{array}$ & $\begin{array}{c}\text { Water } \\
\text { source } \\
\text { by state }\end{array}$ \\
\hline DE-236 & $7 / 26 / 84$ & -12.44 & 306 & Arkansas \\
\hline-237 & $8 / 01 / 84$ & -11.6 & 402 & \\
\hline-238 & $11 / 21 / 83$ & - & 1930 & California \\
\hline-239 & $11^{\prime} / 21 / 83$ & - & 2010 & " \\
\hline-240 & $11 / 19 / 83$ & - & 587 & $"$ \\
\hline-241 & $11 / 21 / 83$ & - & 433 & $"$ \\
\hline-242 & $7^{\prime} / 25 / 85$ & - & - & $"$ \\
\hline-243 & $7 / 29 / 85$ & - & - & $"$ \\
\hline-244 & $7 / 13 / 85$ & - & - & $"$ \\
\hline-245 & $7 / 10 / 84$ & - & - & $"$ \\
\hline-246 & $7 / 10 / 84$ & - & - & $"$ \\
\hline-247 & $7 / 13 / 84$ & - & - & $"$ \\
\hline-248 & $8 / 31 / 83$ & -8.3 & - & Colorado \\
\hline-249 & $10 / 28 / 83$ & -3.8 & - & $"$ \\
\hline-250 & $3 / 24 / 83$ & -6.5 & - & $"$ \\
\hline-251 & $3 / 24 / 83$ & -0.4 & - & $"$ \\
\hline-252 & $3 / 24 / 83$ & -3.9 & - & $"$ \\
\hline-253 & $3 / 24 / 83$ & -4.5 & - & $"$ \\
\hline-254 & $3 / 24 / 83$ & -3.5 & - & $"$ \\
\hline-255 & $3 / 24 / 83$ & -4.7 & - & $"$ \\
\hline-256 & $10 / 9 / 83$ & -4.5 & - & $"$ \\
\hline-257 & $10 / 10 / 83$ & -9.4 & - & $"$ \\
\hline-258 & $8 / 26 / 83$ & -7.1 & - & $"$ \\
\hline-259 & $8 / 30 / 83$ & -3.6 & - & $"$ \\
\hline-260 & $10 / 6 / 83$ & -6.3 & - & $"$ \\
\hline-261 & $10 / 4 / 83$ & -1.3 & - & $"$ \\
\hline-262 & $7 / 18 / 83$ & -9.3 & - & $"$ \\
\hline-263 & $8 / 22 / 83$ & -11.3 & - & $"$ \\
\hline-264 & $3 / 24 / 83$ & -2.5 & - & $"$ \\
\hline-265 & $2 / 7 / 84$ & -10.4 & - & $"$ \\
\hline-266 & $9 / 13 / 84$ & - & 580 & " \\
\hline-267 & $10 / 5 / 83$ & - & - & Indiana \\
\hline-268 & $10 / 5 / 83$ & - & - & " \\
\hline-269 & $10 / 5 / 83$ & - & - & $"$ \\
\hline-270 & $10 / 5 / 83$ & - & - & $"$ \\
\hline-271 & $10 / 5 / 83$ & - & - & $"$ \\
\hline-272 & $10 / 20 / 83$ & - & - & $"$ \\
\hline-273 & $10 / 20 / 83$ & - & - & $"$ \\
\hline-274 & $10 / 20 / 83$ & - & - & $"$ \\
\hline-275 & $10^{\prime} / 19^{\prime} / 83$ & - & - & $"$ \\
\hline-276 & $10 / 19 / 83$ & - & - & $"$ \\
\hline-277 & $11 / 1 / 83$ & - & - & $"$ \\
\hline-278 & $11 / 1 / 83$ & - & - & $"$ \\
\hline-279 & $11 / 1 / 83$ & - & - & $"$ \\
\hline-280 & $10 / 31 / 83$ & - & - & $"$ \\
\hline-281 & $10^{\prime} / 31 / 83$ & - & - & $"$ \\
\hline-282 & $10 / 31 / 83$ & - & - & $"$ \\
\hline-283 & $10 / 31 / 83$ & - & - & $"$ \\
\hline-284 & $10 / 31 / 83$ & - & - & $"$ \\
\hline-285 & $10 / 31 / 83$ & - & - & $"$ \\
\hline-286 & $5 / 8 / 84$ & - & - & $"$ \\
\hline-287 & $10 / 5 / 84$ & - & - & $"$ \\
\hline-288 & $5 / 8 / 84$ & - & - & $"$ \\
\hline-289 & $8 / 30 / 84$ & -27.2 & - & $"$ \\
\hline
\end{tabular}


TABLE 1 (continued)

\begin{tabular}{|c|c|c|c|c|}
\hline $\begin{array}{l}\text { Sample } \\
\text { no. }\end{array}$ & $\begin{array}{l}\text { Colln } \\
\text { date }\end{array}$ & $\begin{array}{c}\delta^{13} \mathrm{C} \\
(\% 0 \mathrm{PDB}) \\
\text { Inorg } \\
\text { carbon }\end{array}$ & $\begin{array}{c}\text { Total } \\
\text { alkalinity } \\
\text { as } \\
\text { bicarbonate } \\
(\mathrm{mg} / \mathrm{L})\end{array}$ & $\begin{array}{c}\text { Water } \\
\text { source } \\
\text { by state }\end{array}$ \\
\hline DE-290 & $7 / 10 / 84$ & - & - & Iowa \\
\hline-291 & $7 / 11 / 84$ & - & - & " \\
\hline-292 & $7 / 9 / 84$ & - & 294 & $"$ \\
\hline-293 & $7 / 7 / 84$ & - & 234 & $"$ \\
\hline-294 & $7 / 11 / 84$ & - & 224 & $"$ \\
\hline-295 & $7 / 11 / 84$ & - & 286 & $"$ \\
\hline-296 & $7 / 6 / 84$ & - & 370 & $"$ \\
\hline-297 & $7 / 6 / 84$ & - & 283 & $"$ \\
\hline-298 & $7 / 5 / 84$ & - & 218 & $"$ \\
\hline-299 & $7 / 5 / 84$ & - & 218 & $"$ \\
\hline-300 & $7 / 3 / 84$ & - & 213 & $"$ \\
\hline-301 & $7 / 3 / 84$ & - & 244 & $"$ \\
\hline-302 & $7 / 3 / 84$ & - & 280 & $"$ \\
\hline-303 & $7 / 6 / 84$ & - & 312 & $"$ \\
\hline-304 & $7 / 12 / 84$ & - & 226 & $"$ \\
\hline-305 & $7 / 9 / 84$ & - & 301 & " \\
\hline-306 & $8 / 3 / 84$ & - & 554 & Missouri \\
\hline-307 & $8 / 1 / 84$ & -12.5 & 332 & " \\
\hline-308 & $7 / 30 / 84$ & -11.9 & 474 & $"$ \\
\hline-309 & $11 / 2 / 83$ & - & 115 & $"$ \\
\hline-310 & $8 / 2 / 84$ & -11.5 & 118 & $"$ \\
\hline-311 & $7 / 31 / 84$ & -13.3 & 167 & $"$ \\
\hline-312 & $10 / 13 / 83$ & -13.2 & - & $"$ \\
\hline-313 & $10 / 27 / 83$ & -14.1 & - & $"$ \\
\hline-314 & $9 / 26 / 84$ & -14.3 & 176 & $"$ \\
\hline-315 & $9 / 25 / 84$ & -14.6 & 85 & $"$ \\
\hline-316 & $10 / 12 / 83$ & -13.1 & - & $"$ \\
\hline-317 & $10 / 14 / 83$ & - & - & $"$ \\
\hline-318 & $10 / 12 / 83$ & -16.6 & - & $"$ \\
\hline-319 & $9 / 26 / 84$ & -13.5 & 131 & $"$ \\
\hline-320 & $10 / 25 / 83$ & -16.1 & - & $"$ \\
\hline-321 & $7 / 26 / 84$ & -13.9 & 276 & $"$ \\
\hline-322 & $7 / 30 / 84$ & -11.6 & 356 & $"$ \\
\hline-323 & $9 / 27 / 84$ & - & 112 & $"$ \\
\hline-324 & $9 / 25 / 84$ & - & 100 & " \\
\hline-325 & $3 / 13 / 84$ & -4.9 & - & Nevada \\
\hline-326 & $5 / 9 / 84$ & -7.5 & - & " \\
\hline-327 & $6 / 16 / 83$ & -5.9 & - & $"$ \\
\hline-328 & $6 / 26 / 84$ & -5.7 & - & $"$ \\
\hline-329 & $4 / 24 / 84$ & -3.5 & - & $"$ \\
\hline-330 & $6 / 25 / 85$ & - & - & $"$ \\
\hline-331 & $6 / 30 / 85$ & - & - & $\prime$ \\
\hline-332 & $6 / 26 / 85$ & - & - & $"$ \\
\hline-333 & $6 / 27 / 85$ & - & - & $"$ \\
\hline-334 & $6 / 28 / 85$ & - & - & $"$ \\
\hline-335 & $6 / 27 / 85$ & - & - & $"$ \\
\hline-336 & $7 / 1 / 85$ & - & - & " \\
\hline
\end{tabular}


TABLE 1 (continued)

\begin{tabular}{|c|c|c|c|c|c|}
\hline \multirow[b]{2}{*}{$\begin{array}{l}\text { Sample } \\
\text { no. }\end{array}$} & \multirow[b]{2}{*}{$\begin{array}{l}\text { Colln } \\
\text { date }\end{array}$} & \multicolumn{2}{|c|}{$\begin{array}{c}\delta^{13} \mathrm{C} \\
(\% \text { PDB })\end{array}$} & \multirow{2}{*}{$\begin{array}{c}\text { Total } \\
\text { alkalinity } \\
\text { as } \\
\text { bicarbonate } \\
(\mathrm{mg} / \mathrm{L})\end{array}$} & \multirow{2}{*}{$\begin{array}{c}\text { Water } \\
\text { source } \\
\text { by state }\end{array}$} \\
\hline & & $\begin{array}{c}\text { Org } \\
\text { carbon }\end{array}$ & $\begin{array}{c}\text { Inorg } \\
\text { carbon }\end{array}$ & & \\
\hline DE-337 & $3 / 14 / 84$ & - & - & - & Nevada \\
\hline-338 & $4 / 22 / 85$ & - & - & - & " \\
\hline-339 & $9 / 30 / 83$ & - & -7.1 & 113 & $" \prime$ \\
\hline-340 & $3 / 13 / 84$ & - & -7.0 & 117 & $"$ \\
\hline-341 & $6 / 13 / 83$ & - & -4.4 & - & $"$ \\
\hline-342 & $6 / 26 / 85$ & - & -12.0 & - & $"$ \\
\hline-343 & $12 / 12 / 83$ & - & - & - & $"$ \\
\hline-344 & $6 / 1 / 84$ & - & - & 112 & $"$ \\
\hline-345 & $7 / 6 / 84$ & - & - & 192 & $"$ \\
\hline-346 & $6 / 20 / 84$ & - & - & - & $"$ \\
\hline-347 & $6 / 20 / 84$ & - & - & 178 & $"$ \\
\hline-348 & $8 / 15 / 84$ & - & - & - & $"$ \\
\hline-349 & $8 / 15 / 84$ & - & - & - & $"$ \\
\hline-350 & $9 / 7 / 84$ & -26.9 & - & - & $"$ \\
\hline-351 & $9 / 7 / 84$ & -24.7 & - & - & " \\
\hline-352 & $9 / 7 / 84$ & -27.1 & - & - & $"$ \\
\hline$-35 \overline{3}$ & $9 / 7 / 84$ & -27.7 & - & - & $"$ \\
\hline-354 & $9 / 7 / 84$ & -24.9 & +1.0 & - & $"$ \\
\hline-355 & $9 / 7 / 84$ & -24.3 & - & - & $"$ \\
\hline-356 & $9 / 7 / 84$ & -23.9 & - & - & $"$ \\
\hline-357 & $9 / 7 / 84$ & -24.1 & - & - & $"$ \\
\hline-358 & $9 / 7 / 84$ & -24.1 & -0.7 & - & $"$ \\
\hline-359 & $9 / 7 / 84$ & -22.5 & -0.3 & - & " \\
\hline-360 & $9 / 7 / 84$ & -27.1 & +1.1 & - & $"$ \\
\hline-361 & $9 / 7 / 84$ & -24.9 & - & - & $"$ \\
\hline-362 & $9 / 7 / 84$ & -24.1 & -2.8 & - & $"$ \\
\hline-363 & $9 / 7 / 84$ & -24.2 & - & - & $"$ \\
\hline-364 & $11 / 30 / 84$ & -22.9 & -1.4 & - & $"$ \\
\hline-365 & $11 / 30 / 84$ & -24.2 & - & - & $"$ \\
\hline-366 & $11 / 30 / 84$ & -22.9 & +0.5 & - & $"$ \\
\hline-367 & $11 / 30 / 84$ & -25.6 & -2.7 & - & $"$ \\
\hline-368 & $6 / 6 / 85$ & -25.0 & -3.8 & - & $"$ \\
\hline-369 & $6 / 6 / 85$ & -22.7 & +2.1 & - & $"$ \\
\hline-370 & $11 / 30 / 84$ & -25.5 & -0.9 & - & $"$ \\
\hline-371 & $11 / 30 / 84$ & -25.9 & +0.0 & - & $"$ \\
\hline-372 & $6 / 6 / 85$ & -23.4 & -0.3 & - & $"$ \\
\hline$-37 \overline{3}$ & $3 / 23 / 84$ & - & -14.7 & 274 & Puerto Rico \\
\hline-374 & $3 / 24 / 84$ & - & -12.9 & 237 & " \\
\hline-375 & $3 / 23 / 84$ & - & -13.6 & 263 & $"$ \\
\hline-376 & $3 / 20 / 84$ & - & -11.6 & 287 & $"$ \\
\hline-377 & $3 / 27 / 84$ & - & -13.4 & 289 & $"$ \\
\hline-378 & $3 / 23 / 84$ & - & -13.3 & 314 & $"$ \\
\hline-379 & $5 / 1 / 84$ & - & -9.45 & 393 & $"$ \\
\hline-380 & $3 / 26 / 84$ & - & -12.2 & 293 & $"$ \\
\hline-381 & $3 / 23 / 84$ & - & -13.1 & 257 & $"$ \\
\hline-382 & $5 / 21 / 85$ & - & -1.8 & 120 & South Carolina \\
\hline-383 & $6 / 26 / 85$ & - & -3.0 & 101 & " \\
\hline-384 & $7 / 23 / 85$ & - & -1.6 & 115 & $"$ \\
\hline-385 & $5 / 22 / 85$ & - & -9.6 & 257 & $"$ \\
\hline-386 & $6 / 25 / 85$ & - & -10.5 & 155 & $"$ \\
\hline
\end{tabular}


TABLE 1 (continued)

\begin{tabular}{|c|c|c|c|c|}
\hline $\begin{array}{l}\text { Sample } \\
\text { no. }\end{array}$ & $\begin{array}{l}\text { Colln } \\
\text { date }\end{array}$ & $\begin{array}{c}\delta^{13} \mathrm{C} \\
(\% 0 \mathrm{PDB}) \\
\text { Inorg } \\
\text { carbon }\end{array}$ & $\begin{array}{c}\text { Total } \\
\text { alkalinity } \\
\text { as } \\
\text { bicarbonate } \\
(\mathrm{mg} / \mathrm{L})\end{array}$ & $\begin{array}{c}\text { Water } \\
\text { source } \\
\text { by state }\end{array}$ \\
\hline $\begin{array}{r}\text { DE-387 } \\
-388 \\
-389 \\
-390 \\
-391 \\
-392 \\
-393 \\
-394 \\
-395 \\
-396 \\
-397 \\
-398 \\
-399 \\
-400 \\
-401 \\
-402 \\
-403 \\
-404 \\
-405 \\
-406 \\
-407\end{array}$ & $\begin{array}{r}8 / 22 / 84 \\
8 / 24 / 84 \\
4 / 25 / 85 \\
4 / 25 / 85 \\
8 / 20 / 84 \\
8 / 28 / 84 \\
4 / 26 / 85 \\
8 / 14 / 84 \\
8 / 30 / 84 \\
7 / 26 / 84 \\
10 / 11 / 83 \\
8 / 17 / 84 \\
8 / 21 / 84 \\
10 / 25 / 84 \\
6 / 19 / 84 \\
6 / 19 / 84 \\
6 / 20 / 84 \\
6 / 6 / 84 \\
6 / 7 / 84 \\
6 / 7 / 84 \\
6 / 21 / 84\end{array}$ & $\begin{array}{c}-20.8 \\
-21.6 \\
-21.0 \\
-20.5 \\
-21.2 \\
-16.8 \\
-19.5 \\
-20.3 \\
-15.1 \\
-12.4 \\
-\overline{17.0} \\
- \\
-5.7 \\
-6.1 \\
-6.0 \\
-7.2 \\
-8.2 \\
-6.0\end{array}$ & $\begin{array}{r}70 \\
39 \\
32 \\
50 \\
66 \\
294 \\
68 \\
66 \\
89 \\
306 \\
755 \\
32 \\
96 \\
754 \\
504 \\
372 \\
494 \\
290 \\
218 \\
363 \\
243\end{array}$ & $\begin{array}{c}\text { Tennessee } \\
\text { " } \\
\text { " } \\
\text { " } \\
\text { " } \\
\text { " } \\
\text { " } \\
\text { " } \\
\text { " } \\
\text { Utah } \\
\text { " } \\
\text { " } \\
\text { " } \\
\text { " } \\
\text { " }\end{array}$ \\
\hline
\end{tabular}

